\title{
Prospective Clinical Application of Thioredoxin Reductase as a Novel Diagnostic Tumor Marker
}

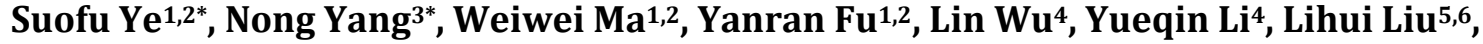 \\ Yi Hui ${ }^{1,2}$, Yu Qiu ${ }^{3}$, Siqing Mei'7, Yan Li ${ }^{7 \#, ~ H u i h u i ~ Z e n g 1,2,4 \# ~}$ \\ ${ }^{1}$ Health Science Center, Peking University, Beijing, China \\ ${ }^{2}$ State Key Laboratory of Natural and Biomimetic Drugs, Peking University, Beijing, China \\ ${ }^{3}$ Department of Medical Oncology, Hunan Provincial Tumor Hospital, Changsha, China \\ ${ }^{4}$ Keaise Clinical Examination Lab, Wuhan, China \\ ${ }^{5}$ Keaise Medicine (Tianjin) Co., Ltd, Tianjin, China \\ ${ }^{6}$ Tianjin International Joint Academy of Biotechnology and Medicine, Tianjin, China \\ ${ }^{7}$ Department of Clinical Laboratory, Renmin Hospital, Wuhan University, Wuhan, China \\ Email: "yanlitf@yahoo.com.cn, \#zenghh@bjmu.edu.cn
}

Received April 2014

\section{Abstract}

Background: Developing a novel, efficient biomarker for detecting malignant tumors is essential for the early diagnosis of cancers. Our aim was to assess the diagnostic value of a potential plasma tumor marker, thioredoxin reductase (TR), which is expressed in many types of malignant tumor, for the non-invasive detection of cancers. Methods: The plasma activities of TR were measured in 1513 patients with common clinical diseases, 59 patients with benign tumors, and 154 patients with cancers and 586 healthy controls. The area under the ROC curve (AUC) of TR and logistic regression results of different groups were compared by sensitivity, specificity and Youden's index. Diagnostic cut-offs and clinical reference intervals were established via ROC curve analysis. Results: The logistic regression indicated that $T R$ activity can discriminate between cancers and benign tumors or other common diseases very well $(p<0.0001)$, with an area under the curve from the receiver-operator characteristics between 0.91 and 0.96 . The positive critical value was 2.51 and the cancer critical value was 9.90. The diagnostic gray zone $(2.51-9.90)$ may be associated with benign tumors and some common clinical diseases. Conclusions: As a novel potential marker of malignant tumors with quantitative evaluation of proliferation, TR activity detection has an excellent diagnostic potential for early-stage malignant tumors. Impact: The convenient, economical, relatively non-invasive, and reproducible detection method of TR activity makes it suitable for routine clinical practice.

\section{Keywords}

Thioredoxin Reductase, Diagnostic Marker, Cancer, Malignant Tumor, TR Activity, Abnormal

\footnotetext{
*Suofu Ye and Nong Yang contributed equally to this work.

"Corresponding authors.
} 


\section{Hyperplasia, Proliferation}

\section{Introduction}

Cancer is the second most common cause of death worldwide, exceeded only by heart disease, and accounts for nearly 1 in every 4 deaths. Development of an efficient biomarker for the early diagnosis of cancer is an important step in reducing mortality from this disease.

Thioredoxin reductase (TR) is a homodimeric flavoenzyme that catalyzes the reduction of thioredoxin (Trx), which is widely expressed in tissue. Several studies have shown that TR is induced in both tumor cells and pre-neoplastic cells [1] [2]. Up-regulation of TR has also been found in lung malignancies such as non-small cell carcinoma [3], malignant mesothelioma [4] [5], glioblastoma multiforme [6], thyroid cancer [7], oral squamous cell carcinoma [8], breast cancer [9], pancreatic cancer [10], prostate cancer [11], hepatocellular carcinoma [12], astrocytic brain tumors [13], and in tumor nodules in a model of hepatocellular carcinogenesis [14]. The up-regulation of TR in tumors could reflect its role in DNA synthesis and in coping with increased oxidative stress, but might also be linked to the regulatory response mediated by p53, indicating that TR is particularly relevant in highly malignant cancers.

Previous studies established the potential of TR as a biomarker in the development of improved diagnostics. Therefore, for the first time, our group applied thioredoxin reductase (TR) detecting method into clinical cancer diagnosis, which was approved by China Food and Drug Administration (CFDA).

It was previously demonstrated that plasma TR activity differs significantly between healthy individuals and those with cancers [15]. However, to date, the plasma TR activity reference intervals for the clinical diagnosis of cancer remains unclear and it is not known if there is an association between plasma TR activities and cancer in patients with gray-zone TR levels.

In the present study, we investigated the plasma TR activities of healthy controls, patients with 12 kinds of common, non-malignant disease, benign tumors, and malignant tumors. We set up a clinical TR reference interval for cancer diagnosis, and determined the cut-off values using a receiver-operator characteristic (ROC) curve, established the diagnostic efficiency, and assessed disease and cancer detection in patients with gray-zone TR levels.

\section{Materials and Methods}

\subsection{Study Groups}

Data on 2,312 subjects between October 2011 and October 2013 were collected from Chinese hospitals and medical center databases: 193 from Beijing, 345 from Tianjin, 849 from Shandong, 755 from Hubei, 110 from Hunan and 60 from Zhejiang. Four groups of subjects were included in the study: 586 [25.35\%] healthy control subjects, 1513 [65.44\%] patients with clinical, non-malignant common diseases, 59 [2.55\%] patients with benign tumors and 154 [6.66\%] patients with cancer. All subjects filled in the informed consent and provided a plasma sample. The study was carried out according to the Helsinki Declaration and approved by the Ethical Committee of Peking University.

\subsection{Control Subjects}

We enrolled control subjects with no smoking, alcoholism or illegal drug use, all of whom were screened by complete physical examination and routine clinical examinations, including electrocardiogram (ECG), standard blood and urine tests, blood biochemistry, chest X-ray film examination, and type-B ultrasound checks.

\subsection{Subjects with Common Non-Malignant Diseases}

The principle inclusion criteria for this group were a clear medical history and imaging results revealing a specific, non-malignant disease. These were hyperglycemia, fatty liver, hypertension, benign prostatic hyperplasia (BPH), uterine fibroids, pure breast hyperplasia, liver cyst, renal cyst, kidney calculi, gallstone, and gallbladder polyp. 


\subsection{Subjects with Benign Tumors}

The clinical diagnosis was confirmed by a CT scan, type-B ultrasound checks, endoscopic exploration, and histopathological examination. The subjects were selected on the basis of presence of benign tumor only, including 24 [40.68\%] benign lumps, 7 [11.86\%] lipoma, 19 [32.20\%] fibroadenoma, and 9 [15.25\%] fibrocystic disease.

\subsection{Subjects with Cancer (Malignant Tumor)}

All the cancers were confirmed by patient history taking, complete physical examination, a CT scan, type-B ultrasound checks, endoscopic exploration, and histopathological and cytological examination. The date of these examinations was considered as the date of cancer diagnosis. Lung cancer, hepatic carcinoma, colorectal cancer, gastric cancer, lymphoma, cervical cancer, cervical metastatic cancer, esophageal cancer, bone cancer, nasopharyngeal carcinoma, breast cancer, kidney cancer, oral cancer, and ovarian cancer cases were all included in this study (Table 1). These subjects were in early stages (stages I and II) and treatment naïve.

\subsection{Sample Processing in the Preanalytical Phase}

Draw the whole blood from the subjects and centrifuge at $3500 \mathrm{rpm} / \mathrm{min}, 5 \mathrm{~min}$. The plasma of subjects was extracted from whole blood and stored at $-20^{\circ} \mathrm{C}$.

\subsection{Measurement of TR Activity}

TR activity was determined spectrophotometrically by monitoring the NADPH-dependent production of 2-nitro5-thiobenzoate (extinction coefficient of $13.600^{-1} \mathrm{~cm}^{-1}$ ) at $412 \mathrm{~nm}$ and at $37^{\circ} \mathrm{C}$. This was achieved using a 1 $1.5 \mathrm{ml}$ plasma sample with the Thioredoxin Reductase (TR) Detection Kit (batch number 2010030, Clairvoyance Health Technology Co., Ltd, Wuhan, China) and a microplate reader (Multiskan Mk3, Thermo Scientific Company), following the manufacturer's instructions. A unit of TR activity was expressed as 1 micromole of NADPH oxidized to NADP+ in one minute under assay conditions.

Sensitivity: the lower limit of detection (LOD) was $1.20 \mathrm{U} / \mathrm{mL}$; the linear range was $1.2-60.0 \mathrm{U} / \mathrm{ml}$. Accuracy: The agreement rate of negative and positive sample groups both reached at $100 \%$. Precision: the coefficients of variability (CV) within group was $8.11 \%$, the $\mathrm{CV}$ between groups was $0 \%$. Stability: under the condition of $37^{\circ} \mathrm{C}$, reagent can be stably stored for 7 days. Thioredoxin Reductase (TR) Detection Kit has been approved by China Food and Drug Administration (Registration number: No. 3400264 2014).

Table 1. Cancer types of recruited subjects.

\begin{tabular}{ccc}
\hline Types of cancers & Number of subjects & Percentage (\%) \\
\hline Lung cancer & 43 & 27.92 \\
Hepatic carcinoma & 12 & 7.79 \\
Colorectal cancer & 10 & 6.49 \\
Gastric cancer & 9 & 5.84 \\
Lymphoma & 9 & 5.84 \\
Cervical cancer & 8 & 5.19 \\
Cervical metastatic cancer & 7 & 4.55 \\
Esophageal cancer & 6 & 3.90 \\
Bone cancer & 5 & 3.25 \\
Nasopharyngeal carcinoma & 12 & 7.79 \\
Breast cancer & 18 & 11.69 \\
Kidney cancer & 4 & 2.60 \\
Oral cancer & 6 & 3.90 \\
Ovarian cancer & 5 & 3.25 \\
Total & 154 & 100 \\
\hline
\end{tabular}




\subsection{Statistical Analysis}

The Statistical Package for Social Sciences 15 (SPSS 15) was used for the statistical analysis of the data. The distribution of plasma TR activity values in the healthy controls, non-malignant disease, benign and cancer groups were assessed using the Kolmogorov-Smirnov test. All values were expressed as the median and interquartile range because the measured data were markedly skewed. The differences between sex groups were analyzed using the non-parametric Mann-Whitney test with the Bonferroni correction for multiple comparisons of age groups. $\mathrm{P}$ values $<0.05$ were considered to be statistically significant. The statistical significance of differences in plasma TR activities between groups was determined by logistic regression. The overall risk (OR) values were calculated to reflect the risk level of a disease developing into a malignancy. To further assess the ability of TR activity to predict cancer, ROC curves, which correlate true and false positive rates (sensitivity and 1-specificity), were constructed, and an area under the curve (AUC) was calculated for each marker. Youden's index (sensitivity + specificity-1) and the cut-off values (the largest Youden's index) were also calculated to set up the clinical reference intervals.

\section{Results}

\subsection{Demographic Data}

The study cohort consisted of 2312 people with 1726 patients and 586 healthy controls, who were divided into groups by sex and age as shown in Table 2. The median-interquartile ranges of TR levels did not differ significantly between males and females $(\mathrm{p}>0.05)$. TR activities in each group were non-normally distributed by the Kolmogorov-Smirnov test $(\mathrm{p}<0.01)$, and non-parametic tests were used in the following statistical analysis.

Table 2. TR activity (U/ml) in different gender and age groups.

\begin{tabular}{|c|c|c|c|c|c|}
\hline Group & $\mathrm{n}$ & $\begin{array}{l}\text { The percentage of } \\
\text { each group (\%) }\end{array}$ & $\begin{array}{l}\text { The percentage of people } \\
\text { with malignant cancer (\%) }\end{array}$ & $\begin{array}{l}\text { Median (IQR) of TR } \\
\text { activity value (U/ml) }\end{array}$ & p-value \\
\hline Gender & $2189^{\mathrm{a}}$ & & & & Wilcoxon-Mann-Whitney test \\
\hline Male & 1116 & 50.98 & 7.17 & $1.20^{\mathrm{C}}(4.90)$ & \\
\hline Female & 1073 & 49.02 & 4.75 & $1.20(4.47)$ & 0.97 \\
\hline Age (years) & $2191^{\mathrm{b}}$ & & & & Bonferroni correction \\
\hline$\leq 30$ & 381 & 17.39 & 2.36 & $1.20(3.25)$ & $\begin{array}{l}1.00 \text { (vs 2) } \\
1.00 \text { (vs 3) } \\
1.00 \text { (vs 3) } \\
0.76 \text { (vs 5) }\end{array}$ \\
\hline $30-40$ & 554 & 25.29 & 1.99 & 1.20 (4.69) & $\begin{array}{l}1.00 \text { (vs } 1) \\
1.00 \text { (vs } 3) \\
1.00 \text { (vs 4) } \\
0.10 \text { (vs 5) }\end{array}$ \\
\hline $40-50$ & 551 & 25.15 & 5.08 & $1.20(4.75)$ & $\begin{array}{l}1.00 \text { (vs } 1) \\
1.00 \text { (vs } 2) \\
1.00 \text { (vs } 4) \\
0.74 \text { (vs 5) }\end{array}$ \\
\hline $50-60$ & 402 & 18.35 & 8.71 & $1.20(5.68)$ & $\begin{array}{l}1.00 \text { (vs } 1) \\
1.00 \text { (vs 2) } \\
1.00 \text { (vs 3) } \\
1.00 \text { (vs 5) }\end{array}$ \\
\hline$\geq 60$ & 303 & 13.83 & 15.51 & $1.20(6.52)$ & $\begin{array}{l}0.76 \text { (vs } 1) \\
0.10 \text { (vs } 2) \\
0.74 \text { (vs } 3) \\
1.00 \text { (vs } 4)\end{array}$ \\
\hline
\end{tabular}

agender information lost in 123 cases; ${ }^{\text {a }}$ age information lost in 121 cases; ${ }^{\mathrm{c}} \mathrm{TR}$ value is equal to 1.2 when the real TR activity of the patient was lower than the detection limits of the TR detection method. 
The median value of TR activity of every age group was $1.20 \mathrm{U} / \mathrm{mL}$, while the interquartile ranges tended to increase with age, indicating the wide distribution of TR activities. However, no age group was found to be significantly different from any others ( $\mathrm{p}>0.05)$.

TR activity in non-malignant disease, and benign and malignant tumors. Logistic regression showed that plasma TR activity was significantly different between healthy controls and patients with hyperglycemia ( $\mathrm{p}<$ $0.05)$, hypertension $(p<0.01)$, liver cysts $(\mathrm{p}<0.05)$, gallstone $(\mathrm{p}<0.05)$, benign tumors $(\mathrm{p}<0.0001)$, and malignant tumors $(\mathrm{p}<0.0001)$ as shown in Table 3.

The OR for the benign and malignant groups were both greater than 1 , as was the $95 \%$ confidence interval of the OR, indicating that TR activity is sufficient to distinguish between healthy controls and those with benign or malignant tumors. The OR values of hyperglycemia, hypertension, liver cysts, gallstones were slightly higher than 1, indicating the diagnostic potential of TR activities in these diseases. As shown in Table 3, the median TR activity $(1.20 \mathrm{U} / \mathrm{mL})$ and the interquartile range $(5.97 \mathrm{U} / \mathrm{mL})$ of the benign group was significantly lower than those of the malignant group (median $=13.78 \mathrm{U} / \mathrm{mL}$, interquartile range $=8.8 \mathrm{U} / \mathrm{mL})(\mathrm{p}<0.01)$. The heterogeneity of tumor specimen may result in a great distribution of TR activities in malignant group; however, a significant higher OR value between malignant tumor group (1.35) compared to the benign tumor group (1.12) suggested its significant diagnostic capability for malignancy.

\subsection{The Diagnostic Efficiency of TR Activity between Malignant and Non-Malignant Disease}

Given the complicated background of patients with various diseases, TR activity should not only be used to distinguish between patients with malignant disease and healthy controls, but also between those with benign tumors or non-malignant disease. Logistic regression analysis indicates that TR activity can be used to discriminate between malignant and benign tumors, as well as between malignant tumors and non-malignant disease ( $\mathrm{p}$ $<0.0001$; Table 4). As shown in Figure 1 and Table 4, the AUCs of TR in malignant and benign tumors or non-malignant disease are $0.91-0.96$, suggesting that TR is statistically significant to distinguish malignant disease from other cases.

Table 3. Diagnostic value of TR activity (U/ml) in different diseases.

\begin{tabular}{|c|c|c|c|c|c|c|}
\hline Disease & $\mathrm{N}$ & Median & IQR & $\mathrm{p}$-value ${ }^{\mathrm{b}}$ & OR & $95 \%$ CI \\
\hline Healthy control & 586 & $1.20^{\mathrm{c}}$ & 2.22 & & & \\
\hline Malignant tumor & 154 & 13.78 & 8.80 & $<0.0001$ & $1.35^{\mathrm{a}}$ & $(1.29,1.41)$ \\
\hline \multicolumn{7}{|c|}{ Non-malignant disease } \\
\hline Hyperglycemia & 37 & 1.20 & 7.02 & 0.0009 & 1.06 & $(1.02,1.09)$ \\
\hline Fatty liver & 416 & 1.20 & 3.25 & 0.19 & 1.02 & $(0.99,1.04)$ \\
\hline Hypertension & 169 & 1.20 & 6.00 & 0.0006 & 1.06 & $(1.03,1.10)$ \\
\hline $\mathrm{BPH}$ & 50 & 1.20 & 5.93 & 0.09 & 1.04 & $(0.99,1.08)$ \\
\hline Benign tumor & 59 & 1.20 & 5.97 & $<0.0001$ & 1.12 & $(1.07,1.17)$ \\
\hline Uterine fibroids & 44 & 1.20 & 2.82 & 1.00 & 1.00 & $(0.94,1.07)$ \\
\hline Pure breast hyperplasia & 405 & 1.20 & 3.62 & 0.22 & 1.02 & $(0.99,1.05)$ \\
\hline Liver cyst & 108 & 1.20 & 5.79 & 0.04 & 1.04 & $(1.00,1.08)$ \\
\hline Renal cyst & 76 & 1.20 & 4.33 & 0.24 & 1.03 & $(0.98,1.07)$ \\
\hline Kidney calculi & 77 & 1.20 & 5.03 & 0.08 & 1.04 & $(1.00,1.08)$ \\
\hline Gallstone & 82 & 1.90 & 5.40 & 0.03 & 1.04 & $(1.01,1.08)$ \\
\hline Gallbladder polyp & 49 & 1.20 & 4.28 & 0.55 & 1.02 & $(0.96,1.07)$ \\
\hline
\end{tabular}

${ }^{\mathrm{a}}$ The OR value of the malignant group was 1.35 , signifying a 1.35 fold increase in risk of developing malignancy if TR activity increases by one unit; ${ }^{\mathrm{b}} \mathrm{P}$ value for logistic regression; ${ }^{\mathrm{C}} \mathrm{TR}$ value is equal to 1.2 when the real TR activity of the patient was lower than the detection limits of the TR detection method. 
The ROC curve of the healthy control vs. non-malignant disease group

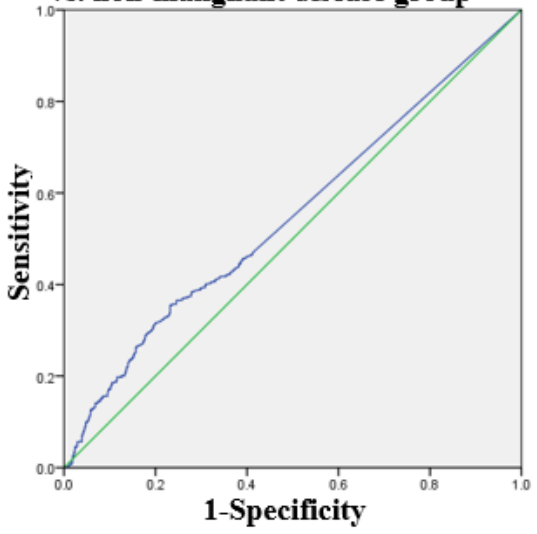

The ROC curve of the benign vs. malignant tumor groups.

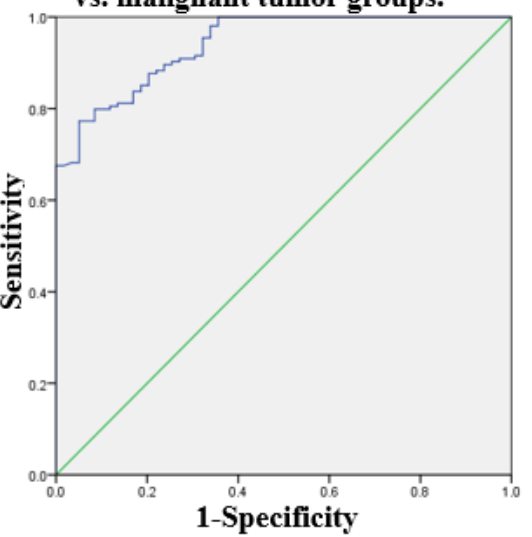

\begin{tabular}{cccccc}
\hline Group & AUC $^{\mathrm{a}}(95 \% \mathrm{CI})$ & Cut-off & $\mathrm{Se}^{\mathrm{b}}$ & $\mathrm{Sp}^{\mathrm{c}}$ & Youden Index \\
\hline $1^{\mathrm{d}}$ & $0.55(0.52,0.58)$ & 2.51 & 0.36 & 0.77 & 0.13 \\
$2^{\mathrm{e}}$ & $0.94(0.91,0.97)$ & 9.90 & 0.78 & 0.95 & 0.73 \\
\hline
\end{tabular}

$\mathrm{a}$, area under curve;

b, sensitivity;

c, specificity

$\mathrm{d}$, the healthy control vs. non-malignant disease group

e, the benign vs. malignant tumor groups

Figure 1. ROC analysis of the healthy control vs. non-malignant disease group, and the benign vs. malignant tumor groups.

Table 4. Diagnostic value of TR activity for malignant tumors.

\begin{tabular}{ccccccccc}
\hline Disease & $\mathrm{P}^{\mathrm{f}}$ & $\mathrm{OR}^{\mathrm{a}}$ & $95 \% \mathrm{CI}^{\mathrm{b}}$ & $\mathrm{AUC}^{\mathrm{c}}(95 \% \mathrm{CI})$ & Cut-off & $\mathrm{Se}^{\mathrm{d}}$ & $\mathrm{Sp}^{\mathrm{e}}$ & Youden Index $^{\text {M }}$ \\
\hline Hyperglycemia & $<0.0001$ & 1.43 & $(1.28,1.61)$ & $0.91(0.86,0.96)$ & 3.48 & 1.00 & 0.62 & 0.62 \\
Fatty liver & $<0.0001$ & 1.24 & $(1.19,1.29)$ & $0.95(0.93,0.96)$ & 3.74 & 0.99 & 0.78 & 0.77 \\
Hypertension & $<0.0001$ & 1.36 & $(1.28,1.45)$ & $0.91(0.88,0.94)$ & 5.37 & 0.91 & 0.73 & 0.64 \\
BPH & $<0.0001$ & 1.42 & $(1.29,1.58)$ & $0.91(0.86,0.96)$ & 6.49 & 0.88 & 0.80 & 0.68 \\
Benign tumor & $<0.0001$ & 1.55 & $(1.37,1.75)$ & $0.94(0.55,0.81)$ & 9.90 & 0.78 & 0.95 & 0.73 \\
Uterine fibroids & $<0.0001$ & 1.67 & $(1.42,1.96)$ & $0.96(0.92,1.00)$ & 4.32 & 0.96 & 0.84 & 0.80 \\
Pure breast hyperplasia & $<0.0001$ & 1.45 & $(1.36,1.54)$ & $0.95(0.94,0.97)$ & 5.03 & 0.92 & 0.86 & 0.78 \\
Liver cyst & $<0.0001$ & 1.40 & $(1.30,1.51)$ & $0.92(0.89,0.96)$ & 6.25 & 0.89 & 0.81 & 0.70 \\
Renal cyst & $<0.0001$ & 1.50 & $(1.33,1.61)$ & $0.93(0.90,0.97)$ & 6.62 & 0.88 & 0.86 & 0.74 \\
Kidney calculi & $<0.0001$ & 1.40 & $(1.29,1.53)$ & $0.92(0.88,0.96)$ & 6.50 & 0.88 & 0.83 & 0.71 \\
Gallstone & $<0.0001$ & 1.47 & $(1.33,1.61)$ & $0.93(0.90,0.96)$ & 6.25 & 0.89 & 0.82 & 0.71 \\
Gallbladder polyp & $<0.0001$ & 1.64 & $(1.42,1.90)$ & $0.96(0.93,0.98)$ & 4.93 & 0.92 & 0.82 & 0.74 \\
\hline
\end{tabular}

${ }^{\mathrm{a}}$ odds ratio; ${ }^{\mathrm{b}}$ confidence interval; ${ }^{\mathrm{c}}$ area under curve; ${ }^{\mathrm{d}}$ sensitivity; ${ }^{\mathrm{e}}$ specificity; ${ }^{\mathrm{f}} \mathrm{P}$ values for logistic regression.

\subsection{Evaluation of TR Diagnostic Parameters}

The sensitivity, specificity, and Youden's index (Table 4) of TR were calculated according to the ROC curves and the stepwise logistic regression. The sensitivities of TR for distinguishing malignant tumors from non-malignant diseases are from 0.78 to 1.00 , among which hyperglycemia revealed the highest sensitivities and benign 
tumors revealed the lowest. The specificity of TR activity ranged between 0.62 and 0.95 , among which benign tumor was the highest and hyperglycemia the lowest. The Youden's indexes ranged between 0.62 and 0.80 with uterine fibroids the highest and hyperglycemia the lowest.

\subsection{TR Diagnostic Intervals and Margins of Various Segments}

In order to provide reasonable reference intervals to clinical diagnosis, the cut-off value of the healthy volunteer versus non-malignant group (2.51) was set as the positive critical value while the cut-off value for the benign versus malignant groups (9.90) was set as the malignant tumor critical value (Figure 1). The interval between the positive critical value and malignant tumor critical value is a clinical diagnostic 'gray area'.

In order to further characterize this diagnostic gray zone, the cut-off values of the non-malignant disease group and malignant tumor group were calculated (Table 4). Results have shown that these cut-off values ranged from 3.48 to 6.62 dependent on various diseases, all of which were lower than the malignant tumor critical value (9.90). These data also suggested that the diagnostic gray zone (2.51 - 9.90) may be associated with high blood pressure, high blood sugar, fatty liver, prostate hyperplasia, breast hyperplasia, uterine fibroids, liver cysts, renal cysts, kidney stones, gallstones, gallbladder polyps, and benign tumors (Figure 2).

\section{Discussion}

TR is an important part of thioredoxin system that is involved in many central intracellular and extracellular processes including cell proliferation, redox regulation of gene expression and signal transduction, protection against oxidative stress, inhibition of apoptosis, growth factor and co-cytokine response, and regulation of the redox state of the extracellular environment [2]. Previous studies suggested that TR expression in tumor cells is ten timeshigher than that in normal tissue [16]. Immunocytochemistry have been used to simultaneously determine the expression and localization of TR in primary human cancers, including breast cancer, thyroid, prostate and colorectal carcinoma, and malignant melanoma, showing greatly over-expressed TR in aggressive tumors with a high proliferation capacity, a low apoptosis rate and an elevated metastatic potential [2]. A correlation between the expression level of TR and degree of differentiation/proliferation has also been observed in human lung cancer [17] and in cell lines derived from mesothelioma [18] [19]. Taken together, these findings strongly suggest an essential role for TR in oncogenesis and hypothesize its potential as a novel marker of a wide range of human malignant tumors, capable of allowing early diagnosis and indicating the level of abnormal hyperplasia.

In this study, the healthy volunteers and patients were randomly enrolled from multiple medical centers on the basis of a comprehensive and integrated examination and accurate diagnosis. Statistical analyses showed no difference in plasma TR activity with respect to sex and age, suggesting that these would not be confounding factors in screening.

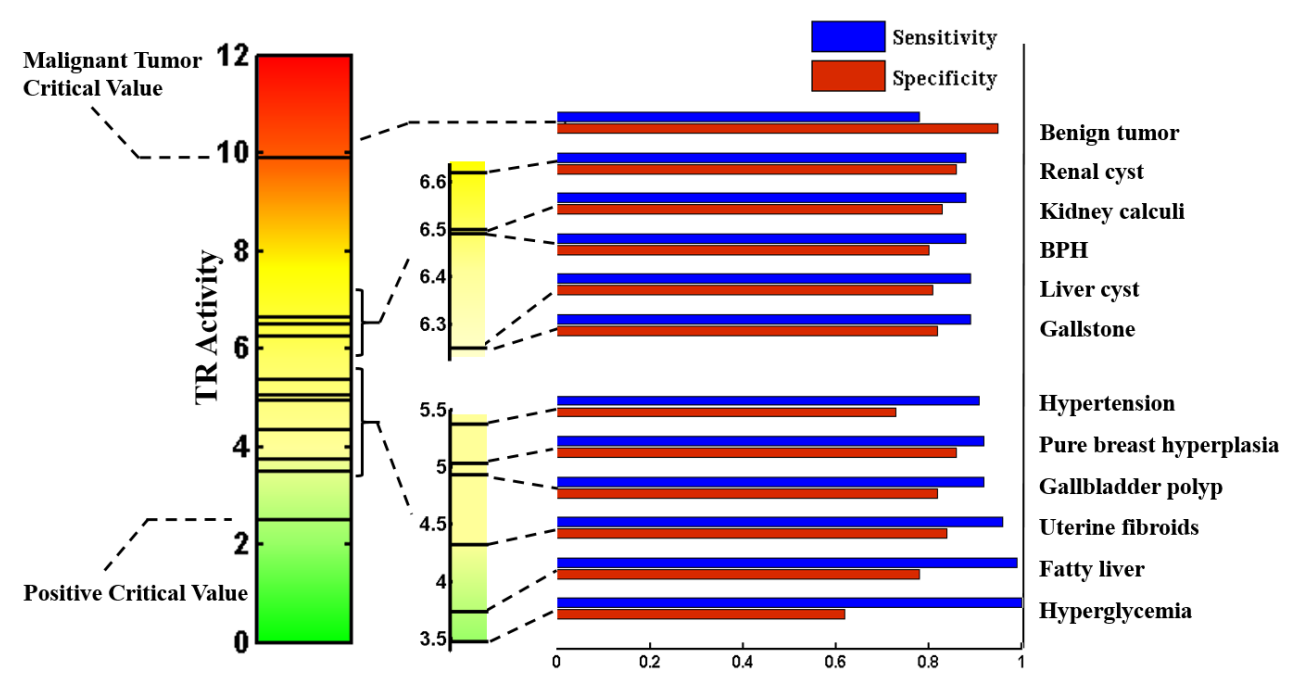

Figure 2. The cut-off values of the non-malignant disease and malignant tumor groups. 
Statistical analyses showed that the median values of TR activity of the healthy control, benign and malignant group was $1.20 \mathrm{U} / \mathrm{mL}, 1.20 \mathrm{U} / \mathrm{mL}$, and $13.78 \mathrm{U} / \mathrm{mL}$, with interquartile ranges of $2.22 \mathrm{U} / \mathrm{mL}, 5.97 \mathrm{U} / \mathrm{mL}$, and $8.80 \mathrm{U} / \mathrm{mL}$, respectively ( $\mathrm{p}<0.01$ ). The wide distribution of TR activities in malignant cases may reflect variations of TR activity in the different cancers.

The plasma TR level was associated with tumor differentiation and proliferation. Hence, similar clinical proliferative diseases and benign tumors may also lead to elevated TR activity. Logistic regression is a multivariate model suitable for comparing the diagnostic validity of tumor markers in patients with different diseases. The logistic regression indicated that TR activity can effectively discriminate between patients with malignant tumors and those with benign tumors or other non-malignant diseases ( $p<0.0001)$. Likewise, the AUCs for TR activity in cases of malignant and benign tumors or non-malignant disease were 0.91 - 0.96, suggesting a high diagnostic potential of TR for malignant tumors, even in the background of other diseases.

The AUC and Youden's index (sensitivity + specificity - 1) reveal diagnostic potential, and help to establish an optimal cut-off value (the highest Youden's index) for distinguishing between malignant and non-malignant cases. The cut-off value to distinguish between the healthy control and non-malignant disease groups was 2.51 , which was set as the positive critical value. The cut-off value for distinguishing between the benign and malignant groups was 9.90, which was set as the malignant tumor critical value. The interval between the positive critical value and malignant tumor critical value is defined as a clinical diagnostic 'gray zone'.

Based on the logistic regression, the OR for the malignant group was 1.35, defining the risk of developing cancer increases 1.35 times if the TR activity increases by one unit, and may indicate that individuals with plasma TR activities exceeding 9.90 are more than 11.03 times $(=1.358)$ more likely to develop cancer. The cutoff values for the non-malignant diseases group and the malignant tumor group ranged from 3.48 to 6.62 , all of which were lower than the malignant tumor critical value (9.90). This suggests that the diagnostic gray zone (2.51 - 9.90) may be associated with high blood pressure, high blood sugar, fatty liver, prostate hyperplasia, breast hyperplasia, uterine fibroids, liver cysts, renal cysts, kidney stones, gallstones, gallbladder polyps, and benign tumors. However, TR activity can significantly discriminate malignant tumor cases from both benign tumors and other non-malignant diseases, suggesting that other clinical backgrounds do not interfere with TRinstructed cancer diagnosis. However, the risk of tumor transformation from benign tumors and other proliferative diseases indicates that this diagnostic gray zone may be of importance in early-stage cancer prognosis.

Apart from the elevated TR values in hyperplasia, a correlation between TR activity and some non-proliferative diseases has also been observed. Previous studies found that Trx and TR mRNA levels were elevated in endothelial cells and macrophages within atherosclerotic plaques, indicating that the thioredoxin system plays an important role in arterial neointima formation during atherosclerosis [20]. It was also found that TR regulates vascular relaxation via antioxidant defense and sGC S-nitrosylation, revealing that TR may be an important target for the treatment of vascular dysfunction and arterial hypertension [21]. The level of TR decreased in vascular smooth muscle cells in vitro when glucose concentration levels were high, primarily due to increased vascular expression of an endogenous inhibitor, thioredoxin-interacting protein (Txnip), and reduced thioredoxin activity, which are normalized by insulin treatment [22]. In this study, we found elevated plasma TR activity in cases of hypertension and hyperglycemia, which were significantly different from those of the healthy control and malignant tumor groups ( $\mathrm{p}<0.001$ ). However, it is not clear how, or even if elevated TR activity contributes to either of these diseases, and the use of TR activity in predicting these diseases requires further validation.

In 2011, the US National Cancer Institute listed 31 tumor markers for cancer diagnosis, among which 17 were detected in the blood. As a novel potential tumor marker in blood, a critical advantage of TR activity is that it can predict cancers with a sensitivity of 78\% - 100\%, much higher than commonly used clinical tumor markers, such as CEA (sensitivity 14\% - 72.9\% in different cancers) [23]-[30], AFP (sensitivity 25\% - 72.1\%) [31], and CA125 (sensitivity 3.1\% - 68.2\%) [24] [26] [32]. Moreover, by indicating the level of proliferation, TR activity has great potential to predict tumorigenesis at an earlier stage for a wider range of cancers.

The World Health Organization considers that more than $40 \%$ of cancers are preventable [33]. Thus, cancer prevention with early-stage screening is becoming increasingly important. As a novel potential marker of malignant tumors with quantitative evaluation of proliferation, TR activity detection has an excellent diagnostic potential for early-stage malignant tumors. Furthermore, this convenient, economical, relatively non-invasive, and reproducible detection method of TR activity makes it suitable for routine clinical practice. 


\section{Acknowledgments}

This work was supported in part by the National Natural Science Foundation [81372266].

\section{Conflict of Interest Statement}

We declare that we have no financial and personal relationships with other people or organizations that can inappropriately influence our work, there is no professional or other personal interest of any nature or kind in any product, service and/or company that could be construed as influencing the position presented in, or the review of, the manuscript entitled.

\section{References}

[1] Berggren, M., Gallegos, A., Gasdaska, J.R., Gasdaska, P.Y., Warneke, J. and Powis, G. (1996) Thioredoxin and Thioredoxin Reductase Gene Expression in Human Tumors and Cell Lines, and the Effects of Serum Stimulation and Hypoxia. Anticancer Research, 16, 3459-3466.

[2] Lincoln, D.T., Emadi, E.M.A., Tonissen, K.F. and Clarke, F.M. (2003) The Thioredoxin-Thioredoxin Reductase System: Over-Expression in Human Cancer. Anticancer Research, 23, 2425-2433.

[3] Soini, Y., Kahlos, K., Napankangas, U., Kaarteenaho-Wiik, R., Saily, M., Koistinen, P., et al. (2001) Widespread Expression of Thioredoxin and Thioredoxin Reductase in Non-Small Cell Lung Carcinoma. Clinical Cancer Research, 7, 1750-1757.

[4] Nguyen, P., Awwad, R.T., Smart, D.D.K., Spitz, D.R. and Gius, D. (2006) Thioredoxin Reductase as a Novel Molecular Target for Cancer Therapy. Cancer Letters, 236, 164-174. http://dx.doi.org/10.1016/j.canlet.2005.04.028

[5] Kahlos, K., Soini, Y., Saily, M., Koistinen, P., Kakko, S., Paakko, P., et al. (2001) Up-Regulation of Thioredoxin and Thioredoxin Reductase in Human Malignant Pleural Mesothelioma. International Journal of Cancer, 95, 198-204. http://dx.doi.org/10.1002/1097-0215(20010520)95:3<198::AID-IJC1034>3.0.CO;2-F

[6] Kemerdere, R., Kacira, T., Hanimoglu, H., Kucur, M., Tanriverdi, T. and Canbaz, B. (2013) Tissue and Plasma Thioredoxin Reductase Expressions in Patients with Glioblastoma Multiforme. Journal of Neurological Surgery Part ACentral European Neurosurgery, 74, 234-238.

[7] Lincoln, D.T., Al-Yatama, F., Mohammed, F.M.A., Al-Banaw, A.G., Al-Bader, M., Burge, M., et al. (2010) Thioredoxin and Thioredoxin Reductase Expression in Thyroid Cancer Depends on Tumour Aggressiveness. Anticancer Research, 30, 767-775.

[8] Iwasawa, S., Yamano, Y., Takiguchi, Y., Tanzawa, H., Tatsumi, K. and Uzawa, K. (2011) Upregulation of Thioredoxin Reductase 1 in Human Oral Squamous Cell Carcinoma. Oncology Reports, 25, 637-644..

[9] Cadenas, C., Franckenstein, D., Schmidt, M., Gehrmann, M., Hermes, M., Geppert, B., et al. (2010) Role of Thioredoxin Reductase 1 and Thioredoxin Interacting Protein in Prognosis of Breast Cancer. Breast Cancer Research, 12.

[10] Yan, C., Shieh, B., Reigan, P., Zhang, Z., Colucci, M.A., Chilloux, A., et al. (2009) Potent Activity of Indolequinones against Human Pancreatic Cancer: Identification of Thioredoxin Reductase as a Potential Target. Molecular Pharmacology, 76, 163-172.

[11] Singh, S.S., Li, Y., Ford III, O.H., Wrzosek, C.S., Mehedint, D.C., Titus, M.A., et al. (2008) Thioredoxin Reductase 1 Expression and Castration-Recurrent Growth of Prostate Cancer. Translational Oncology, 1, 153-157.

[12] Cunnea, P., Fernandes, A.P., Capitanio, A., Eken, S., Spyrou, G. and Bjornstedt, M. (2007) Increased Expression of Specific Thioredoxin Family Proteins; A Pilot Immunohistochemical Study on Human Hepatocellular Carcinoma. International Journal of Immunopathology and Pharmacology, 20, 17-24.

[13] Haapasalo, H., Kylaniemi, M., Paunu, N., Kinnula, V.L. and Soini, Y. (2003) Expression of Antioxidant Enzymes in Astrocytic Brain Tumors. Brain Pathology, 13, 155-164.

[14] Biaglow, J.E. and Miller, R.A. (2005) The Thioredoxin Reductase/Thioredoxin System—Novel Redox Targets for Cancer Therapy. Cancer Biology \& Therapy, 4, 6-13.

[15] Luo, Y., Ma, W.W., Yang, J., Wu, L., Li, Y.Q., Zhao, Y.Y. and Zeng, H.H. (2012) Clinical Value of Thioredoxin Reductase Detection Kit for Diagnosis of Cancers. International Journal of Laboratory Medicine, 33, 13.

[16] Zhang, X.T. and Xiong, Y.M. (2004) The Progress in Studies of Thioredoxin Reductase. Foreign Medical Sciences-Section of Medgeography, 25, 4.

[17] Fernandes, A.P., Capitanio, A., Selenius, M., Brodin, O., Rundlof, A.K. and Bjornstedt, M. (2009) Expression Profiles of Thioredoxin Family Proteins in Human Lung Cancer Tissue: Correlation with Proliferation and Differentiation. Histopathology, 55, 313-320. 
[18] Sun, X.J., Dobra, K., Bjornstedt, M. and Hjerpe, A. (2000) Upregulation of 9 Genes, Including That for Thioredoxin, during Epithelial Differentiation of Mesothelioma Cells. Differentiation, 66, 181-188.

[19] Rundlof, A.-K., Fernandes, A.P., Selenius, M., Babic, M., Shariatgorji, M., Nilsonne, G., et al. (2007) Quantification of Alternative mRNA Species and Identification of Thioredoxin Reductase 1 Isoforms in Human Tumor Cells. Differentiation, 75, 123-132.

[20] Takagi, Y., Gon, Y., Todaka, T., Nozaki, K., Nishiyama, A., Sono, H., et al. (1998) Expression of Thioredoxin Is Enhanced in Atherosclerotic Plaques and during Neointima Formation in Rat Arteries. Laboratory Investigation, 78, 957966.

[21] Choi, H., Tostes, R.C. and Webb, R.C. (2011) Thioredoxin Reductase Inhibition Reduces Relaxation by Increasing Oxidative Stress and S-Nitrosylation in Mouse Aorta. Journal of Cardiovascular Pharmacology, 58, 522-527.

[22] Schulze, P.C., Yoshioka, J., Takahashi, T., He, Z.H., King, G.L. and Lee, R.T. (2004) Hyperglycemia Promotes Oxidative Stress through Inhibition of Thioredoxin Function by Thioredoxin-Interacting Protein. Journal of Biological Chemistry, 279, 30369-30374.

[23] Yamamoto, H., Miyake, Y., Noura, S., Ogawa, M., Yasui, M., Ikenaga, M., et al. (2001) Tumor Markers for Colorectal Cancer. Gan to Kagaku Ryoho Cancer \& Chemotherapy, 28, 1299-1305.

[24] Tamakoshi, K., Kikkawa, F., Shibata, K., Tomoda, K., Obata, N.H., Wakahara, F., et al. (1996) Clinical Value of CA125, CA19-9, CEA, CA72-4, and TPA in Borderline Ovarian Tumor. Gynecologic Oncology, 62, 67-72.

[25] Ni, X.G., Bai, X.F., Mao, Y.L., Shao, Y.F., Wu, J.X., Shan, Y., et al. (2005) The Clinical Value of Serum CEA, CA19-9, and CA242 in the Diagnosis and Prognosis of Pancreatic Cancer. Ejso, 31, 164-169.

[26] Ma, S., Shen, L., Qian, N. and Chen, K. (2011) The Prognostic Values of CA125, CA19.9, NSE, and SCC for Stage I NSCLC Are Limited. Cancer Biomarkers, 10, 155-162.

[27] Imada, T., Rino, Y., Takahashi, M., Hatori, S., Shiozawa, M., Tanaka, J., et al. (1997) Expression of CA19-9, SLX, STN and CEA in Relatively Early Gastric Carcinoma. Oncology Reports, 4, 899-904.

[28] Huang, M.-S., Jong, S.-B., Tsai, M.-S., Lin, M.-S., Chong, I.-W., Lin, H.-C., et al. (1996) Comparison of Cytokeratin Fragment 19 (CYFRA 21-1), Tissue Polypeptide Antigen (TPA) and Carcinoembryonic Antigen (CEA) as Tumor Markers in Lung Cancer. European Journal of Cancer, 32A.

[29] Hanagiri, T., Sugaya, M., Takenaka, M., Oka, S., Baba, T., Shigematsu, Y., et al. (2011) Preoperative CYFRA 21-1 and CEA as Prognostic Factors in Patients with Stage I Non-Small Cell Lung Cancer. Lung Cancer, 74, 112-117.

[30] Changchine, C.S., Yung, C.Y. and Tzen, K.Y. (1991) Serum Carcinoembryonic Antigen (CEA) and Carbohydrate Antigen (CA 19-9) Values in Patients with Pancreatic Cancer or Pancreatitis. Chang Gung Medical Journal, 14, 32-38.

[31] Yao, F., Guo, J.M., Xu, C.F., Lou, Y.L., Xiao, B.X., Zhou, W.H., et al. (2005) Detecting AFP mRIVA in Peripheral Blood of the Patients with Hepatocellular Carcinoma, Liver Cirrhosis and Hepatitis. Clinica Chimica Acta, 361, 119127.

[32] Takeshima, N., Shimizu, Y., Umezawa, S., Hirai, Y., Chen, J.T., Fujimoto, I., et al. (1994) Combined Assay of Serum Levels of CA125 and CA19-9 in Endometrial Carcinoma. Gynecologic Oncology, 54, 321-326.

[33] Danaei, G., Vander Hoorn, S., Lopez, A.D., Murray, C.J.L. and Ezzati, M. (2005) Comparative Risk Assessment C. Causes of Cancer in the World: Comparative Risk Assessment of Nine Behavioural and Environmental Risk Factors. Lancet, 366, 1784-1793. 